\title{
Penicillin-Binding Protein 2x of Streptococcus pneumoniae: The Mutation Ala707Asp Within the C-terminal PASTA2 Domain Leads to Destabilization
}

\author{
Inga Schweizer, ${ }^{*}$ Katharina Peters, ${ }^{*}$ Christoph Stahlmann, ${ }^{\dagger}$ Regine Hakenbeck, ${ }^{\star}$ and Dalia Denapaite
}

Streptococcus pneumoniae penicillin-binding protein $2 \mathrm{x}(\mathrm{PBP} 2 \mathrm{x})$ is an enzyme involved in the last stages of peptidoglycan assembly and essential for bacterial growth and survival. PBP2x localizes to the division site, a process that depends on its Penicillin-Binding Protein And Serine-Threonine-kinase Associated (PASTA) domains, which was previously demonstrated via GFP-PBP $2 x$ in living cells. During this study a mutant strain was isolated in which the GFP-PBP2x fusion protein did not localize at division sites and it contained reduced amounts of the full-length GFP-PBP2x. We now show that this defect is due to a point mutation within the C-terminal PASTA2 domain of PBP2x. The mutant protein was analyzed in detail in terms of beta-lactam binding, functionality, and localization in live cells. We demonstrate that the mutation affects the GFP-tagged PBP2x variant severely and renders it susceptible to the protease/chaperone HtrA.

\section{Introduction}

$\mathbf{P}$ ENICILLIN-BINDING PROTEINS (PBPs) are targets for B-lactam antibiotics. They are membrane-associated enzymes implicated in the last steps of peptidoglycan (PG) biosynthesis, a major component of the bacterial cell wall. Streptococcus pneumoniae contains six PBPs. ${ }^{9}$ The high molecular weight (hmw) PBPs are subdivided into two classes based on the architecture of their domains (for reviews, see Goffin and Ghuysen, ${ }^{6}$ Sauvage et al. ${ }^{32}$; Zapun et $a l .{ }^{38}$ ). Members of class A (S. pneumoniae PBP1a, PBP1b, and PBP2a) are bifunctional enzymes containing transglycosylase and transpeptidase (TP) domains. The two $S$. pneumoniae class B PBPs (PBP2x and PBP2b) are monofunctional TPs and individually essential in $S$. pneumoniae. ${ }^{2,13}$ Each of the class A hmw PBPs can be deleted in $S$. pneumoniae, but pbplapbp2a double mutants are not viable. ${ }^{12,27}$ The low-molecularweight PBP3 acts as D,D-carboxypeptidase. ${ }^{8}$

During cell division, all S. pneumoniae hmw PBPs are localized at mid-cell where cell wall synthesis occurs by a combination of peripheral and septal PG synthesis. $2,20,34,39$ PBP2 $x$ is involved in septal and PBP2b in peripheral synthesis. $^{2,39}$ The septal localization of PBP2x in S. pneumoniae has been demonstrated by immunofluorescence microscopy and by GFP-tagging in living cells. ${ }^{15,24,25,29}$
S. pneumoniae $\mathrm{PBP} 2 \mathrm{x}$ was the first hmw $\mathrm{PBP}$ whose crystal structure was solved. ${ }^{28}$ The structure of a soluble PBP $2 x$ derivative revealed a three-domain organization composed of an elongated, "sugar tongue"-like N-terminal domain, a central TP domain and a C-terminal extension attached to the TP domain via a long flexible linker region. In addition, $\mathrm{PBP} 2 \mathrm{x}$ contains an $\mathrm{N}$-terminal cytoplasmic tail of 27 amino acids (aa) and a single membrane spanning segment. The N-terminal domain of unknown function is located adjacent to the TP domain and may serve as a pedestal, placing the catalytic region of the protein away from the cell membrane and toward the PG. ${ }^{17}$ The TP domain has an active site reminiscent of class $A$ and $C \beta$ lactamases and harbors three conserved aa motifs with the active site $\operatorname{Ser}_{337}$ residue, which forms a covalent complex with beta-lactams. Interestingly, the X-ray structure of an acylated PBP2x in complex with cefuroxime shows the presence of two antibiotic molecules, one as expected covalently attached to $\mathrm{Ser}_{337}$, and another sandwiched between the TP domain and the first of two homologous C-terminal noncatalytic domains. ${ }^{7}$ These domains are named PenicillinBinding Protein And Serine-Threonine-kinase Āssociated (PASTA) domains since they exist in single or multiple copies in some hmw PBPs and in bacterial Ser/Thr kinases. ${ }^{37}$ In $S$. pneumoniae the only PBP that harbors PASTA domains is

Department of Microbiology, University of Kaiserslautern, Kaiserslautern, Germany.

†Present affiliation: Department of Experimental Neurology, Saarland University, Homburg, Germany.

Present affiliation: Stiftung Alfried Krupp Kolleg Greifswald, Greifswald, Germany.

*Both authors contributed equally to this work. 
PBP2x. Each PASTA domain of $\sim 70$ aa consists of three $\beta$ sheets and one $\alpha$-helix, with a loop region of variable length between the first and second $\beta$-sheets. These structural elements are highly conserved among PASTA subunits of different proteins although their sequence identity is only $\sim 10 \%$. $^{37}$

Since the structures of $\beta$-lactam antibiotics mimic the terminal portion of the PG stem peptide, it has been suggested that PASTA motifs might bind noncross-linked PG, the substrate of PBPs. ${ }^{4,37}$ Indeed, this has been verified for several Ser/Thr kinases. ${ }^{18,23,33,35}$ Unfortunately, no specific binding sites have been identified in the PASTA domain of different Ser/Thr kinases. Recent studies with PonA2, a key PBP from Mycobacterium tuberculosis, show that its PASTA domain is not able to bind noncross-linked PG, $\beta$-lactams, or polymeric PG. ${ }^{3}$ These results indicate that the role of PASTA domains cannot be generalized.

Little is known about the PASTA domains of $S$. pneumoniae PBP2x. Deletion of the last 40 aa of PASTA2 strongly affects beta-lactam binding at the active site, whereas deletion of 30 aa does not. ${ }^{22}$ In addition, it was recently shown that septal localization of PBP2x is driven by its PASTA domains. ${ }^{29}$ During this work a mutant was isolated that did not localize at septal sites although it contained a full-length GFP-PBP2x fusion protein albeit at reduced amount. We now show that this defect is due to a mutation localized in the PASTA2 domain of PBP2x. The mutant protein was analyzed in detail in terms of functionality, beta-lactam binding and localization in live cells.

\section{Materials and Methods}

\section{Bacterial strains, plasmids, and growth conditions}

All strains and plasmids used in this work are listed in Table 1. S. pneumoniae strains were grown at $30^{\circ} \mathrm{C}$ or $37^{\circ} \mathrm{C}$ in complex $\mathrm{C}$ medium ${ }^{14}$ supplemented with $0.1 \%$ yeast extract $(\mathrm{C}+\mathrm{Y}) . \mathrm{D}$-agar ${ }^{1}$ supplemented with $3 \%$ defibrinated sheep blood was used to grow $S$. pneumoniae on plates. To induce expression of $g f p$ fusions under the $\mathrm{P}_{\mathrm{Zn}}$ promoter, $0.15 \mathrm{mM} \mathrm{ZnCl}_{2}$ was added to the $\mathrm{C}+\mathrm{Y}$ medium. Growth in liquid culture was monitored by nephelometry (nephelo units, $[\mathrm{N}]$ ).

Escherichia coli strain DH5 $\alpha$ was used as a host for cloning and propagation of plasmids. E. coli strains were grown at $37^{\circ} \mathrm{C}$ either in luria broth (LB) medium with aer- ation or on LB agar plates. ${ }^{31}$ Growth of E. coli was followed by measuring the optical density at $600 \mathrm{~nm}$.

\section{Transformation}

Transformation of S. pneumoniae R6 and derivatives was carried out as described previously. ${ }^{19}$ The transformation efficiency was determined using chromosomal DNA of $S$. pneumoniae AmiA9 conferring resistance to streptomycin ${ }^{30}$ and was calculated as the percentage of transformants on selective plates (colony forming units [CFU]) compared to CFU on control plates without selective antibiotic. S. pneumoniae transformants were selected by plating on D-agar supplemented with $3 \%$ defibrinated sheep blood and $2.5 \mu \mathrm{g} /$ $\mathrm{ml}$ tetracycline or $20 \mu \mathrm{g} / \mathrm{ml}$ spectinomycin.

E. coli was transformed according to Hanahan ${ }^{11}$ and transformants were selected in the presence of $100 \mu \mathrm{g} / \mathrm{ml}$ ampicillin.

\section{DNA manipulations and construction of strains and plasmids}

Procedures such as DNA isolation, restriction, ligation, and agarose gel electrophoresis were performed as recently depicted. ${ }^{29}$ DNA modifying enzymes were purchased from New England Biolabs or Fermentas (Thermo Scientific) and used as described by the manufacturer.

To construct the plasmid pFP098, a polymerase chain reaction (PCR) fragment was amplified from strain DKL04 with the primers pbp2x_gfp_f/pbp2x_gfp_r ${ }^{29}$ using iProof high-fidelity DNA polymerase (Bio-Rad Laboratories). The amplified $p b p 2 x$ fragment was digested with NotI and SpeI and inserted into the same sites in pJWV25, ${ }^{5}$ generating the plasmid pFP098.

The plasmid pFP098 was transformed into S. pneumoniae R6 strain using the tetracycline resistance marker tet $M$ for selection, generating strain DKL22. Correct integration into the $\operatorname{bgaA}$ region via double-crossover was confirmed by PCR amplification with the oligonucleotides bga_check_F and bga_check_R as well as DNA sequencing. ${ }^{29}$ To exclude the possibility that any mutation occurred in the $p b p 2 x$ gene, the $g f p-p b p 2 x$ fusion was amplified and sequenced.

To delete the PBP2x gene, the plasmid $\mathrm{p} 2 \mathrm{xKO}^{29}$ was transformed into competent DKL04 cells and transformants were selected with spectinomycin in the presence of $\mathrm{ZnCl}_{2}$

Table 1. Bacterial Strains and Plasmids Used in This Study

\begin{tabular}{|c|c|c|}
\hline \multicolumn{3}{|l|}{ Strains } \\
\hline R6 & Unencapsulated, nonvirulent descendent of D39 & 16,26 \\
\hline DKL03 & $\mathrm{R} 6, \Delta$ bgaA::tetM-P $\mathrm{P}_{\mathrm{Zn}}-g f p-p b p 2 x ; \mathrm{Tet}^{\mathrm{R}}$ & 29 \\
\hline DKL04 & $\mathrm{R} 6, \Delta$ bgaA::tetM- $\mathrm{P}_{\mathrm{Zn}}-g f p-p b p 2 x_{\mathrm{A} 707 \mathrm{D}} \mathrm{G} 749 \mathrm{~V} ; \mathrm{Tet}^{\mathrm{R}}$ & This study \\
\hline DKL041 & DKL04, but $\Delta b g a A:: t e t M-\mathrm{P}_{\mathrm{Zn}}-g f p-p b p 2 x_{\mathrm{G} 749 \mathrm{~V}} ; \Delta p b p 2 x:: a a d 9 ; \mathrm{Spc}^{\mathrm{R}}$ & This study \\
\hline DKL22 & $\mathrm{R} 6, \Delta$ bgaA::tetM- $\mathrm{P}_{\mathrm{Zn}}-g f p-p b p 2 \mathrm{x}_{\mathrm{A} 707 \mathrm{D}} ; \mathrm{Tet}^{\mathrm{R}}$ & This study \\
\hline DKL22 $\Delta h t r A$ & DKL22, but $\Delta h t r A ; \operatorname{Kan}^{r}$ & This study \\
\hline \multicolumn{3}{|l|}{ Plasmids } \\
\hline pJWV25 & pPP2 derivative, ${ }^{10}$ carries $\mathrm{P}_{\mathrm{Zn}^{-}} g f p^{+}$, Amp, ${ }^{\mathrm{R}}$ Tet $^{\mathrm{R}}$ & 5 \\
\hline pFP098 & pJWV25 derivative, carries $\mathrm{P}_{\mathrm{Zn}}-g f p-p b p 2 x_{\mathrm{A} 707 \mathrm{D}}$ fusion & This study \\
\hline $\mathrm{p} 2 \mathrm{xKO}$ & $\begin{array}{l}\text { pUC19 derivative carrying an insert composed of Spc resistance } \\
\text { cassette and upstream/downstream flanking regions of } p b p 2 x ; \mathrm{Amp}^{\mathrm{R}}\end{array}$ & 29 \\
\hline
\end{tabular}

\footnotetext{
${ }^{\mathrm{a} A n t i b i o t i c ~ r e s i s t a n c e ~ m a r k e r s: ~ T e t, ~ t e t r a c y c l i n e ; ~ S p c, ~ s p e c t i n o m y c i n ; ~ K a n, ~ k a n a m y c i n ; ~ A m p, ~ a m p i c i l l i n . ~}$
} 
$(0.15 \mathrm{mM})$. Correct integration into the genome was verified by PCR and sequencing.

To construct an htrA deletion strain, a PCR cassette replacing $h t r A$ with the kanamycin resistance gene aphIII was inserted into the genome of R6 derivatives as described previously. $^{29}$

\section{Labeling and detection of PBPs}

Preparation of samples, PBP labeling of cell lysates with Bocillin $^{\mathrm{TM}} \mathrm{FL}$ and separation of proteins by sodium dodecyl sulfate polyacrylamide gel electrophoresis (SDS-PAGE) was carried out as described recently. ${ }^{29}$

\section{Western blot analysis}

Cells from exponentially growing cultures were collected at a cell density of $\mathrm{N}=70-80$ by centrifugation. Whole cell lysates were obtained by incubating cells in the presence of $0.2 \%$ Triton $\mathrm{X}-100$ for $30 \mathrm{~min}$ at $37^{\circ} \mathrm{C}$; per sample the cell equivalent of $1.5 \mathrm{ml}$ at $\mathrm{N}=20$ was used. After SDS-PAGE, proteins were transferred onto a PVDF membrane (Roche Diagnostics) and detected with affinity-purified polyclonal PBP $2 x,{ }^{21}$ or anti-GFP antibodies (polyclonal rabbit antibodies; Invitrogen). After incubation with the secondary antibody (alkaline phosphatase-conjugated goat anti rabbit immunoglobulin G; Sigma-Aldrich) staining was conducted with 4-nitrobluetetrazolium chloride and 5-bromo-4-chloro3-indolylphosphate (Roche). Immunoblots were quantified using the programme Image $\mathrm{J}(\mathrm{NIH})$.

\section{Fluorescence microscopy}

Fluorescence microscopy was performed as previously described. ${ }^{29}$ Briefly, cells grown in liquid medium at $30^{\circ} \mathrm{C}$ were analyzed by phase contrast or epi-fluorescence microscopy using an Eclipse E600 (Nikon) microscope and $100 \times$ NA 1.4 oil immersion objective; pictures were taken with a DXM1200C camera (Nikon). Fluorescence signals of GFP were visualized with the Epi-FL filterblock B-2E/C (EX: 465-495, DM: 505, BA: 515-555; Nikon) using identical fluorescence intensity. Typical exposure times were between 1 and $2 \mathrm{sec}$. Image analysis was carried out using Nikon Imaging software Nis-Elements BR.

\section{Results}

\section{Identification of mutations in PASTA2 of GFP-PBP2x}

In a recent report we described the construction of an N-terminal GFP-PBP2x fusion protein for localization studies in live cells ${ }^{29}$ using the vector pJWV25. It harbors the zinc-inducible $\mathrm{P}_{\mathrm{Zn}}$ promotor and integrates in the chromosome of $S$. pneumoniae by double-crossover at the $b g a A$ locus. ${ }^{5}$ Such constructs were readily obtained, and most of them showed localization of GFP-PBP2x at septal sites as shown for DKL03. ${ }^{29}$ However, one transformant was obtained during the screening procedure in which the fluorescence signal was dispersed throughout the cytoplasm. Sequencing of $g f p-p b p 2 x$ in this particular transformant named DKL04 revealed that it contains two mutations, both of which were located within the PASTA2 domain of GFP-PBP2x

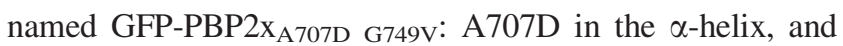
G749V at the very end of $\beta 3$-sheet (Fig. 1).

The localization pattern of GFP-PBP2x is dependent on the presence of the two C-terminal PASTA domains of PBP2x. ${ }^{29}$ Moreover, Maurer et al. have shown that deletion of the last 40 aa in PBP2x resulted in an almost complete loss of beta-lactam binding, that is, the presence of the $\alpha$ helix of PASTA2 domain appears to be critical for antibiotic binding, whereas deletion of 30 C-terminal aa had no functional consequences. ${ }^{22}$ Therefore, we analyzed the ef-

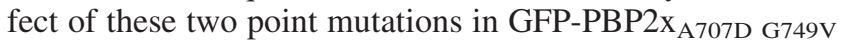
on beta-lactam binding and localization in detail.

\section{Localization and beta-lactam binding of GFP-PBP2 $2 x_{A 707 D}$ G749V}

The expression of GFP-PBP2 $\mathrm{x}_{\mathrm{A} 707 \mathrm{D} \text { G749V } \text { was induced }}$ by addition of $\mathrm{ZnCl}_{2}$ to the growth medium. First, we localized the GFP-PBP2 $x_{\text {A707D G749V }}$ in DKL04 strain by fluorescence microscopy. The protein did not localize to mid-cell and the fluorescence signal was distributed throughout the cytoplasm. However, a small fraction of the cells ( 3 out of 480 examined cells) showed septal localization (see arrow in Fig. 2A) and will be discussed below.

Next, Bocillin FL binding of GFP-PBP2 $x_{\text {A707D G749V was }}$ examined in cell lysates by fluorography (Fig. 2B, top). Surprisingly, only a thin band with the correct size was

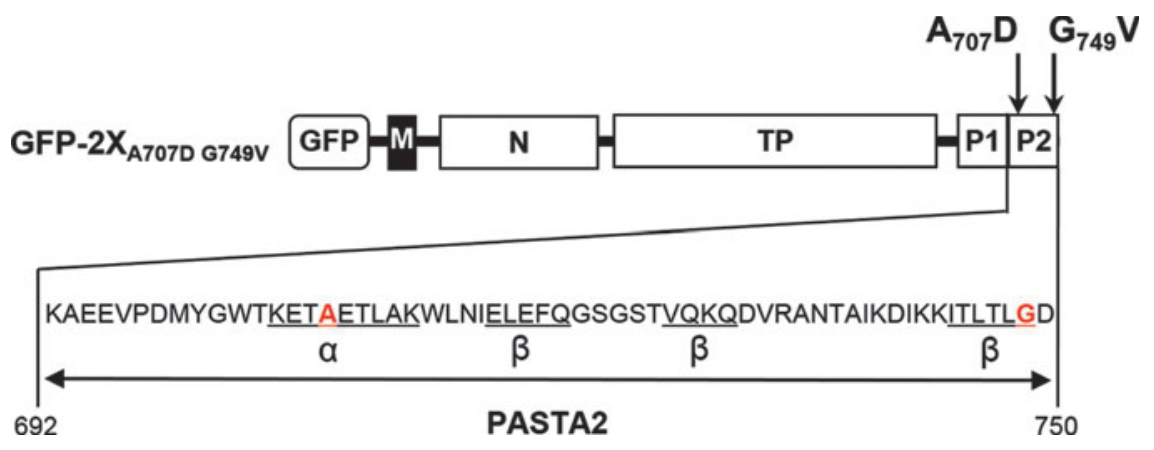

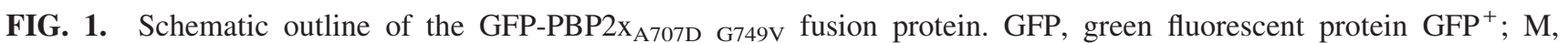
transmembrane helix; N, N-terminal domain; TP, transpeptidase/penicillin-binding domain; P1 and P2, C-terminal PASTA domains. Structural features of the P2 domain are shown in detail below: $\alpha$ : alpha-helix and $\beta$ : beta-sheets are underlined according to ${ }^{37}$; first and last aa of P2 are indicated. ${ }^{7}$ The mutations in GFP-PBP2 $x_{\text {A707D G749V }}$ (DKL04) are marked in red.

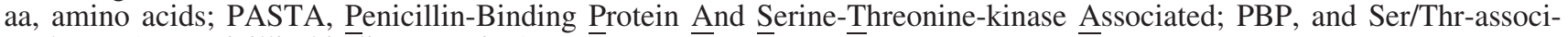
ated; PBP2x, penicillin-binding protein $2 \mathrm{x}$. 

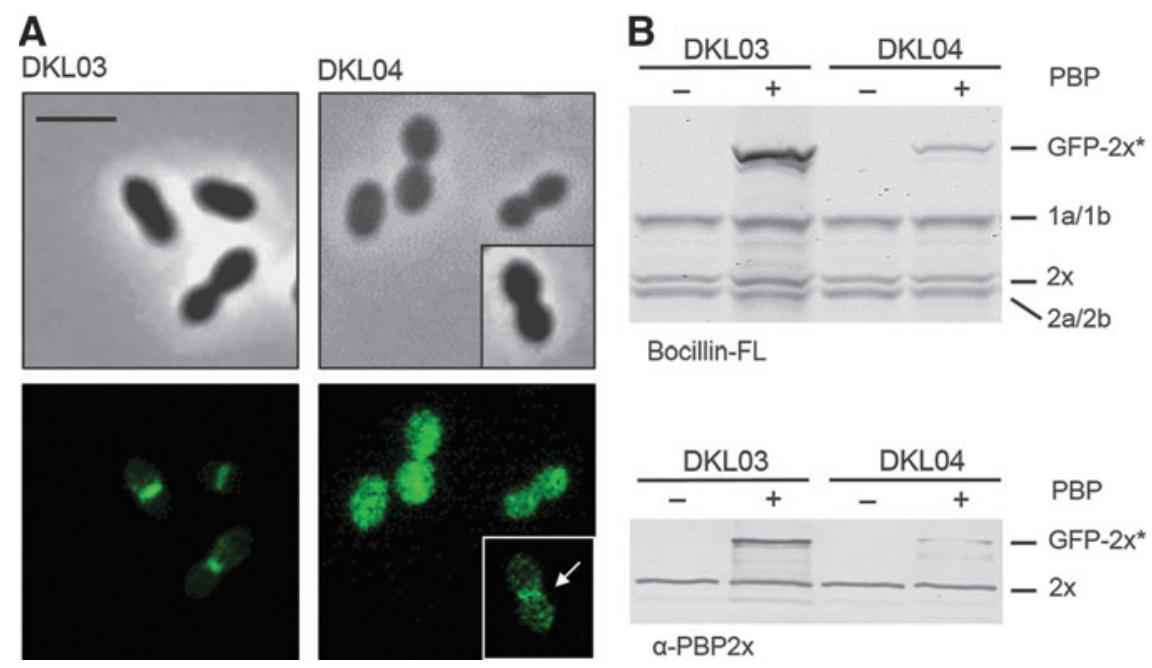

FIG. 2. Localization and production of the GFP-PBP2 $\mathrm{x}_{\mathrm{A} 707 \mathrm{D}} \mathrm{G} 749 \mathrm{~V}$ fusion protein in DKL04. (A) DKL04 (R6, $\mathrm{P}_{\mathrm{Zn}}-g f p$ $p b p 2 x_{\mathrm{A} 707 \mathrm{D}}$ G749V) and the control strain DKL03 (R6, $\mathrm{P}_{\mathrm{Zn}^{-}} g f p-p b p 2 x$ ) were grown in the presence of $\mathrm{ZnCl} \mathrm{C}_{2}$. Top: Phase contrast microscopy; Bottom: Fluorescence signal. Micrograph inserts show a cell with septal localization (see arrow), which occurs on rare occasions in DKL04 (3 out of 480 counted cells). Scale bar $=2 \mu \mathrm{m}$. (B) Top: PBP profiles in strains DKL03 and DKL04. Cell lysates were incubated with Bocillin ${ }^{\mathrm{TM}}$ FL and PBPs visualized after separation by SDS-PAGE followed by fluorography. Bottom: Western blot developed with anti-PBP2x $(\alpha-\mathrm{PBP} 2 \mathrm{x})$ antibodies. + and - indicate growth with and without $\mathrm{ZnCl}_{2}$. The positions of PBPs and GFP-2x* (GFP-PBP2x and GFP-PBP2x $\mathrm{A}_{\mathrm{A} 07 \mathrm{D}}$ G749V) are indicated on the right. SDS-PAGE, sodium dodecyl sulfate-polyacrylamide gel electrophoresis.

detectable in the DKL04 lysate, whereas the GFP-PBP2x band was perfectly present in the lysates of the control strain DKL03. Western blot analyses of cell lysates using antiPBP2x antibodies showed that the GFP-PBP2 $x_{A 707 D}$ G749V fusion protein is also only present in small amounts in DKL04 (Fig. 2B, bottom). The amount of GFP-PBP2 $x_{\text {A707D }}$ G749V was more than 10-fold lower when compared with the wild type GFP-PBP2x. In this context it should be noted that the fusion protein GFP-PBP2x in the control strain DKL03 was present in slightly higher amounts compared with native PBP2x. ${ }^{29}$

Taken together these data indicate that the GFPPBP2 $x_{\text {A707D G749V }}$ is not stable and subject to degradation. Alternatively, it is possible that at least one of the mutations is not tolerated by the cell, and that the highly reduced number of cells with septal fluorescence signal is the product of revertants that can easily arise by recombination with the native $p b p 2 x$.

\section{The mutation A707 is not tolerated in GFP-PBP2x}

To test whether GFP-PBP2 $x_{A 707 D}$ G749V can replace the native PBP2x gene, we tried to delete the native copy of $p b p 2 x$ in DKL04 using the plasmid $\mathrm{p} 2 \mathrm{xKO} .{ }^{29}$ This is readily possible in the control strain DKL03 where such transformants were obtained at high frequency, confirming that the GFP-PBP2x fusion is fully functional. ${ }^{29}$ In contrast, the transformation efficiency in DKL04 as recipient was below $1 \%$ compared to the DKL03 control (Table 2). DNA sequencing of two such transformants revealed that indeed the native $p b p 2 x$ was deleted. However, the GFP-PBP $2 x$ fusion protein carried only the mutation V749, whereas in both cases the other mutation D707 had reverted to A707. This strongly suggests that D707 severely affects at least the function of the protein, whereas this is not the case for V749.
One transformant, DKL041, was used for further experiments. As can be seen in Fig. 3A, the GFP-PBP $2 x_{G 749 V}$ fusion was produced in clearly higher amounts than in the parental strain DKL04, and was active in Bocillin FL binding. It should be noted that a very thin band corresponding to GFP-PBP $2 \mathrm{x}_{\mathrm{G} 749 \mathrm{~V}}$ protein was detected in cell lysates of DKL041 prepared from cultures grown in the absence of $\mathrm{ZnCl}_{2}$ (Fig. 3A). This probably represents some residual GFP-PBP2 $\mathrm{x}_{\mathrm{G} 749 \mathrm{~V}}$ originating from the starting culture grown with $\mathrm{ZnCl}_{2}$ since it takes a considerable time before the fusion protein is depleted upon shift to zinc-free medium. ${ }^{29}$ Moreover, the GFP-PBP $2 \mathrm{x}_{\mathrm{G} 749 \mathrm{~V}}$ perfectly localized at the division septum (Fig. 3B).

To exclude the possibility that other mutations are involved in these phenotypes, three independent transformants obtained with a PCR product covering the GFP-PBP2 $\mathrm{x}_{\mathrm{A} 707 \mathrm{D} \text { G749V }}$ gene were produced. All three transformants showed the same phenotype as described above. Taken together these data indicate that the mutation V749 is well tolerated by the cells and has no apparent effect on PBP2x properties. In other words, it is the mutation D707 that affects the PBP2x protein, that is, the

TABle 2. Transformation EfFiciency

\begin{tabular}{lcc}
\hline & \multicolumn{2}{c}{ Transformation efficiency } \\
\cline { 2 - 3 } Strain & AmiA9 $^{\mathrm{a}}$ & $p 2 \times K O^{\mathrm{b}}$ \\
\hline DKL03 & 0.50 & 1.65 \\
DKL04 & 0.46 & 0.01 \\
DKL22 & 0.53 & 0.02 \\
\hline
\end{tabular}

${ }^{\mathrm{a}}$ Chromosomal donor DNA from Streptococcus pneumoniae AmiA9 was used and $\mathrm{Str}^{\mathrm{R}}$ transformants were selected.

${ }^{\mathrm{b}}$ Spectinomycin-resistant transformants were selected using the plasmid $\mathrm{p} 2 \mathrm{xKO}$. 


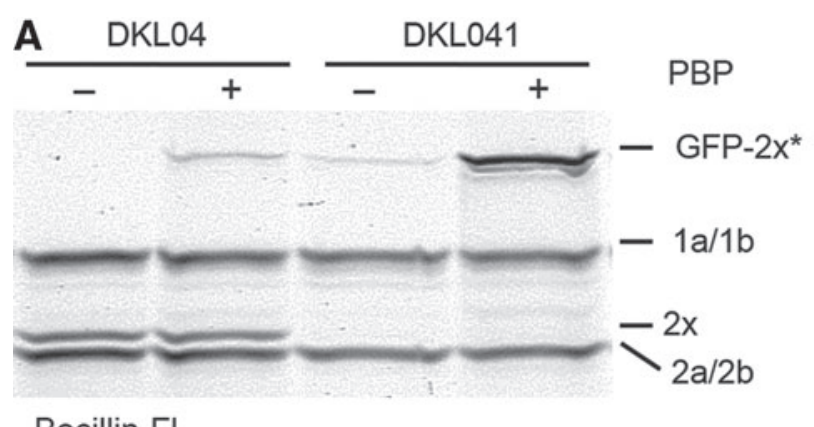

B

Bocillin-FL
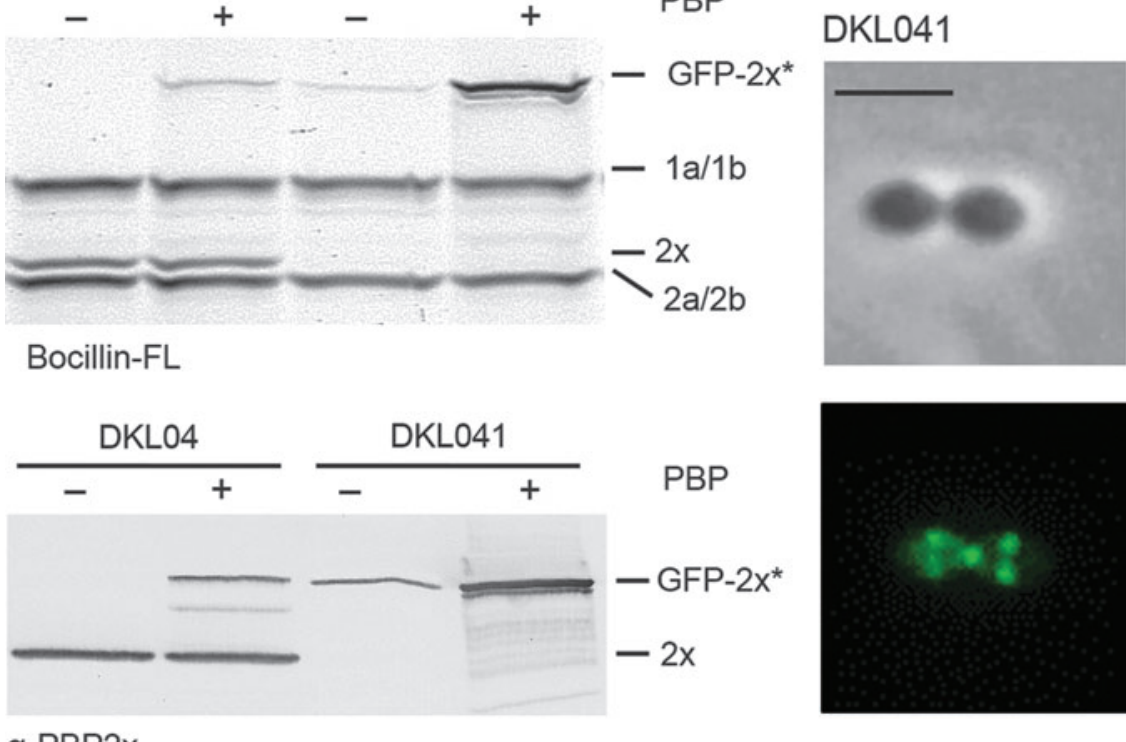

a-PBP2x

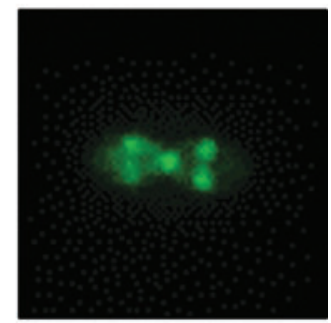

FIG. 3. Cellular localization of the GFP-fusion protein in DKL041. (A) Top: PBP profile analysis. PBPs in cell lysates were labeled with Bocillin ${ }^{\mathrm{TM}}$ FL and visualized by fluorography. Bottom: Western blot developed with anti-PBP2x anti-

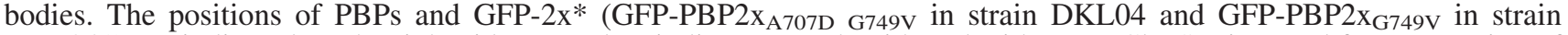
DKL041) are indicated on the right side. + and - indicate growth with and without $\mathrm{ZnCl}_{2}$. Strains used for preparation of cell lysates are indicated on top. (B) Localization of GFP-PBP2 $\mathrm{x}_{\mathrm{G} 749 \mathrm{~V}}$ in DKL041 strain. Top: Phase contrast microscopy; Bottom: Fluorescence signal. Scale bar $=2 \mu \mathrm{m}$.

position A707 in the $\alpha$-helix of PASTA2 is critical for stability and thus affects localization and function of PBP2x as well. In this context it should be noted that revertants of DKL04 expressing GFP-PBP2 $\mathrm{x}_{\mathrm{G} 749 \mathrm{~V}}$ occur at low frequency, probably via recombination with the native $p b p 2 x$. Thus, it is possible that the small amount of GFP-PBP2x seen in DKL04 is due to such revertants already present in the culture.

\section{Ala707 is important for PBP2x functionality}

Finally, we constructed the mutant DKL22 that expresses a GFP-PBP2x containing the single mutation D707 to prove that it is indeed this mutation that confers the phenotype observed in DKL04. Upon induction of $g f p-p b p 2 x_{\mathrm{A} 707 \mathrm{D}}$ in DKL 22 by $\mathrm{ZnCl}_{2}$, cell lysates were incubated with Bocillin FL for visualization of PBPs and PBP2x was specifically labeled with anti-PBP2x antibodies after western blotting as shown above. Only a thin band was visible in the lysate of strain DKL22 on the fluorogram at the position of the GFPPBP2x (Fig. 4A, top), and the presence of very low amounts of the GFP-PBP2 $\mathrm{x}_{\text {A707D }}$ fusion protein in DKL22 when compared with DKL03 was confirmed immunologically (Fig. 4A, bottom). The fluorescence signal in DKL22 was dispersed throughout the cells and did not localize at the septum (Fig. 4B), similar to the results obtained with DKL04. Furthermore, we tried to delete the native copy of pbp $2 x$ in DKL22 and the transformants were obtained with similar transformation efficiency as in the case of DKL04 (Table 2). The analysis of one transformant revealed that indeed the native $p b p 2 x$ was deleted but the D707 mutation had reverted to A707. As expected, the GFP-PBP2x fusion in these revertants perfectly localized at the division septum (data not shown). These experiments confirm that the mu- tation D707 located within the $\alpha$-helix of PASTA2 severely affects PBP2x functionality.

\section{GFP-PBP $2 x_{A 707 D}$ is targeted by HtrA}

We have recently shown that derivatives of GFP-PBP $2 x$ containing various deletions are degraded by the serine protease/chaperon HtrA, and most of them did not localize to septal sites in the presence of HtrA. Therefore, GFPPBP $2 \mathrm{x}_{\mathrm{A} 707 \mathrm{D}}$ was compared in DKL22 $\Delta$ trA to that in the parental DKL22. Western blot analysis using anti-GFP antibodies demonstrated that indeed the fusion protein GFPPBP2 $x_{A 707 D}$ was degraded in DKL22 (i.e., in the presence of HtrA; Fig. 5A), whereas the degradation products were barely visible in the HtrA deletion mutant (see arrows in Fig. 5A). With anti-PBP2x antibodies another degradation product of the fusion protein was detected in HtrA deletion strain that was already seen on western blot developed with anti-GFP antibodies (see arrow in Fig. 5B). Moreover, DKL22 $2 h t r A$ contained a GFP-PBP2 $\mathrm{x}_{\text {A707D }}$ protein active in Bocillin ${ }^{\mathrm{TM}} \mathrm{FL}$ binding, which is hardly visible in DKL22 (Fig. 5C).

Fluorescence microscopy showed that deletion of htrA affected the localization of GFP-PBP $2 \mathrm{x}_{\mathrm{A} 707 \mathrm{D}}$ fusion protein. The percentage of cells showing fluorescence signals at septal sites increased from $13 \%$ (DKL22) to $35 \%$ in DKL22 $\Delta h t r A$. In most cells it still localized in the membrane, at cell poles or in irregular patches (Fig. 5D).

\section{Discussion}

The present study extends previous observations related to in vivo localization studies of PBP $2 \mathrm{x}$, one of the two essential PBPs in S. pneumoniae, which is an important component of 


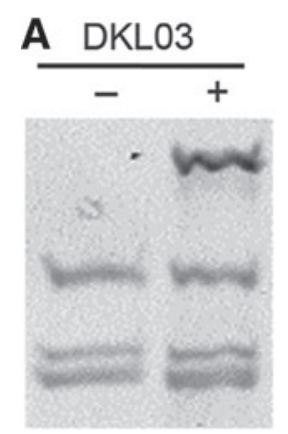

Bocillin-FL
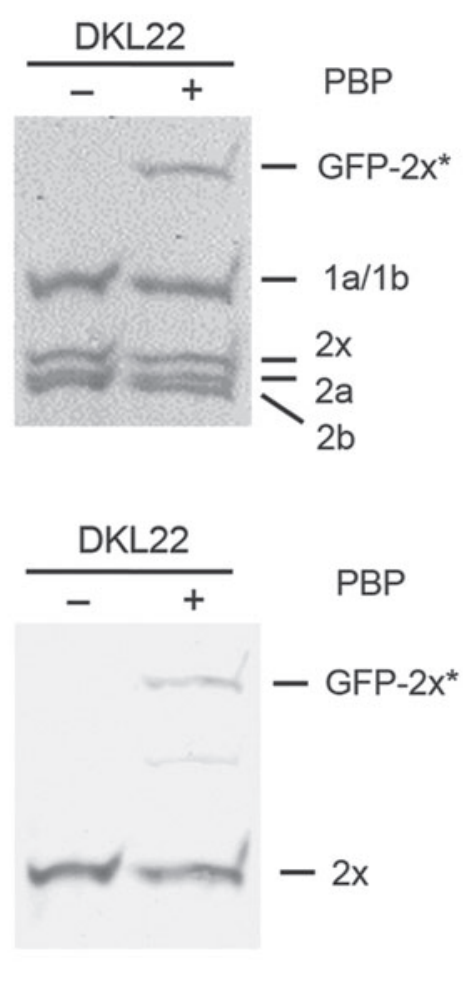

\section{B}

DKL22
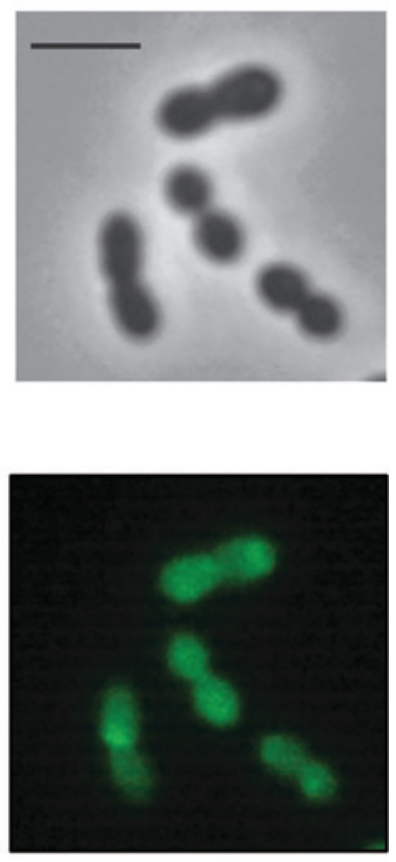

FIG. 4. Effect of the mutation D707. (A) Top. PBP in cell lysates of strains DKL03 (R6, $\mathrm{P}_{\mathrm{Zn}}$-gfp-pbp2x) and DKL22 (R6, $\left.\mathrm{P}_{\mathrm{Zn}}-g f p-p b p 2 x_{\mathrm{A} 707 \mathrm{D}}\right)$ were labeled with Bocillin FL and visualized by fluorography. Bottom: Western blot of the gel on the top developed with anti-PBP2x antibodies. + and - indicate growth with and without $\mathrm{ZnCl}_{2}$. The positions of the PBPs and GFP-2x* (GFP-PBP2x and GFP-PBP2 $x_{\text {A707D }}$ ) are indicated on the right. Strains used for preparation of cell lysates are indicated on top. (B) Micrographs of strain DKL22 grown in the presence of $\mathrm{ZnCl}_{2}$. Top: Phase contrast microscopy; Bottom: Fluorescence signal. Scale bar $=2 \mu \mathrm{m}$.
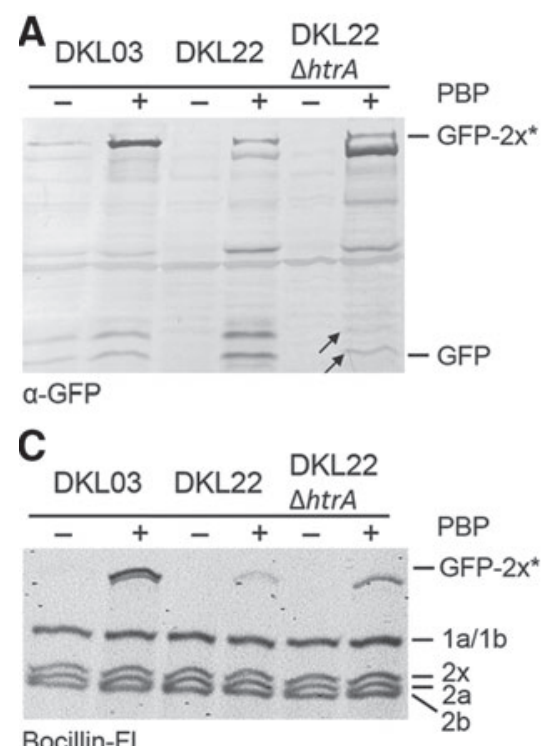

B

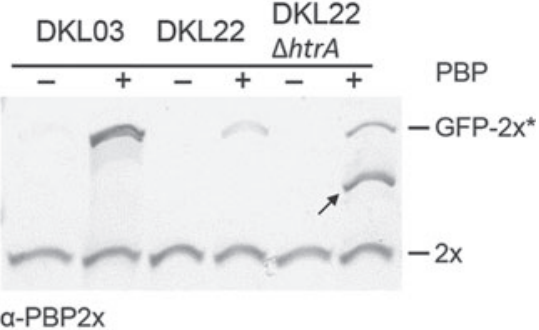

D DKL22AhtrA
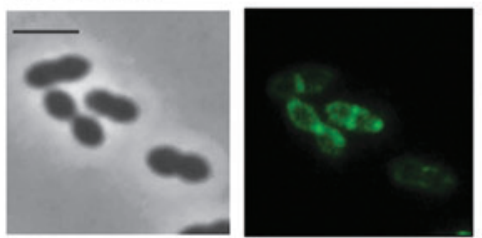

FIG. 5. The fusion protein GFP-PBP2 $\mathrm{x}_{\mathrm{A} 707 \mathrm{D}}$ is a target of HtrA. The protein GFP-PBP2 $\mathrm{x}_{\mathrm{A} 707 \mathrm{D}}$ (DKL22) was analyzed in presence or absence of HtrA. (A) Western blot analysis using anti-GFP ( $\alpha$-GFP) antibodies. The positions of GFP-2x* $\left(\right.$ GFP-PBP2x and GFP-PBP2 $\left.x_{\mathrm{A} 707 \mathrm{D}}\right)$ are indicated on the right. Cells were grown in the presence or absence of $\mathrm{ZnCl}_{2}$ (indicated + and - ). Arrows point to degradation products that are barely visible in case of $\Delta h t r A$. (B) Western blot of cell lysates developed with anti-PBP2x antibodies. + and - : growth with and without $\mathrm{ZnCl}_{2}$. The position of GFP-2x* and PBP2x are marked. Arrow: product seen in $\Delta h t r A$ background. (C) PBP profile analysis. PBPs in cell lysates were labeled with Bocillin FL and visualized by fluorography after separation by SDS-PAGE. On the right the positions of PBPs are indicated. Strains as indicated on top were grown in the presence $(+)$ or absence $(-)$ of $\mathrm{ZnCl}_{2}$. (D) Localization of GFPPBP2 $\mathrm{x}_{\mathrm{A} 707 \mathrm{D}}$ in the absence of HtrA. DKL22 $\Delta h t r A$ was grown in the presence of $\mathrm{ZnCl}_{2}$. Left: Phase contrast microscopy; Right: Fluorescence signal. Scale bar $=2 \mu \mathrm{m}$. 
the cell division apparatus, and moreover a major determinant for beta-lactam resistance. Using GFP-PBP2x fusion proteins we showed in a previous study that the PASTA domains are required for proper localization at septal sites, and that GFPPBP2x derivatives with various deletions are targeted and degraded by the serine protease/chaperon HtrA. ${ }^{29}$ We now show that also a single aa exchange A707D within the PASTA2 domain has severe effects in different respects. First, the GFPPBP2 $\mathrm{x}_{\mathrm{A} 707 \mathrm{D}}$ mutant protein did not localize at septal sites (Fig. 4B). Second, deletion of the genomic wild-type $p b p 2 x$ was not possible in strain DKL22, and the amount of GFP-PBP2 $x_{\text {A707D }}$ was severely reduced. On the other hand, GFP-PBP2 $\mathrm{x}_{\mathrm{A} 707 \mathrm{D}}$ was detectable in cells where $h t r A$ was deleted, and degradation products, which were clearly visible in the wild-type genetic background, were hardly detectable any more. Thus, the mutation renders A707D the protein recognizable by HtrA, strongly suggesting a severe structural impact.

This is the first case where a single point mutation in the C-terminal PASTA domain is shown to have a severe effect on PBP2x. So far only mutations within the TP domain have been described that mediate resistance against beta-lactams (for review, see Hakenbeck et al. ${ }^{9}$ ), and at least some of them apparently affect the physiology of the cell as well. ${ }^{19,40}$ It has been discussed that the PASTA domains are mutational hotspots, and that the mutations consistently occur at the same sites, whereas the remaining sites are conserved. ${ }^{4,37}$ The position A707 is highly conserved in PBP2x of S. pneumoniae and the closely related species Streptococcus mitis including mosaic variants of resistant strains; only in a few Streptococcus oralis strains the alteration V707 occurs, an aa, which contains also a small hydrophobic side chain (own unpublished data). This is in agreement with our hypothesis that the position A707 is of structural and/or functional importance.

The mutant isolated initially contained two mutations: A707D and G749V. PBP2x is 750 aa long, and since deletion of the C-terminal 30 aa are tolerated without any impact on beta-lactam binding, ${ }^{22}$ it is unlikely that G749V drastically affects function and/or stability of the protein, especially since no large or charged side chains are introduced by this mutation. Moreover, the side chain is oriented toward the surface of the protein and thus has probably little impact on its structure. In fact, in a mutant containing only GFP-PBP $2 \mathrm{x}_{\mathrm{G} 749 \mathrm{~V}}$ the protein appears to localize at the septum and to bind Bocillin FL similar to the wild-type PBP2x (Fig. 5B), confirming that there is no detectable effect of this mutation on the functionality of PBP2x. On the other hand, the mutation A707D introduces a large charged side chain, which is directed toward the interior of the C-terminal domain and might well affect its tertiary structure severely and thereby attracting HtrA. On the other hand, GFP-PBP2 $\mathrm{x}_{\mathrm{G} 749 \mathrm{~V}}$ was perfectly able to bind Bocillin FL as shown in DKL22 2 htrA (Fig. 5C). Thus, this mutant protein differs from the deletion derivatives described recently, ${ }^{29}$ in that its enzymatic function in terms of beta-lactam binding and thus PG synthesis appears not to be affected, whereas localization at the septum and thus its function in the division process is severely hampered. This might indirectly affect the interaction with other components of the divisome complex, which appears to be governed by HtrA, which itself localizes at septal sites. ${ }^{36}$ Future studies will help to unravel the interactive network of divisome components.

\section{Acknowledgments}

This work was supported by a grant from the Deutsche Forschungsgemeinschaft Ha 1011/11-1.

\section{Disclosure Statement}

No competing financial interests exist.

\section{References}

1. Alloing, G., C. Granadel, D.A. Morrison, and J.-P. Claverys. 1996. Competence pheromone, oligopeptide permease, and induction of competence in Streptococcus pneumoniae. Mol. Microbiol. 21:471-478.

2. Berg, K.H., G.A. Stamsas, D. Straume, and L.S. Havarstein. 2013. The effect of low Pbp2b levels on cell morphology and peptidoglycan composition in Streptococcus pneumoniae R6. J. Bacteriol. 195:4342-4354.

3. Calvanese, L., L. Falcigno, C. Maglione, D. Marasco, A. Ruggiero, F. Squeglia, R. Berisio, and G. D'Auria. 2013. Structural and binding properties of the PASTA domain of PonA2, a key penicillin binding protein from Mycobacterium tuberculosis. Biopolymers 101:712-719.

4. Dessen, A., N. Mouz, E. Gordon, J. Hopkins, and O. Dideberg. 2001. Crystal structure of PBP2x from a highly penicillin-resistant Streptococcus pneumoniae clinical isolate: a mosaic framework containing 83 mutations. J. Biol. Chem. 276:45106-45112.

5. Eberhardt, A., L.J. Wu, J. Errington, W. Vollmer, and J.W. Veening. 2009. Cellular localization of cholineutilization proteins in Streptococcus pneumoniae using novel fluorescent reporter systems. Mol. Microbiol. 74:395-408.

6. Goffin, C., and J.-M. Ghuysen. 2002. Biochemistry and comparative genomics of SxxK superfamily acyltransferases offer a clue to the mycobacterial paradox: presence of penicillin-susceptible target proteins versus lack of efficiency of penicillin as therapeutic agent. Microbiol. Mol. Biol. Rev. 66:706-738.

7. Gordon, E., N. Mouz, E. Duee, and O. Dideberg. 2000. The crystal structure of the penicillin-binding protein $2 \mathrm{x}$ from Streptococcus pneumoniae and its acyl-enzyme form: implication in drug resistance. J. Mol. Biol. 299:477-485.

8. Hakenbeck, R., and M. Kohiyama. 1982. Purification of penicillin-binding protein 3 from Streptococcus pneumoniae. Eur. J. Biochem. 127:231-236.

9. Hakenbeck, R., R. Brückner, D. Denapaite, and P. Maurer. 2012. Molecular mechanism of beta-lactam resistance in Streptococcus pneumoniae. Future Microbiol. 7:395-410.

10. Halfmann, A., R. Hakenbeck, and R. Brückner. 2007. A new integrative reporter plasmid for Streptococcus pneumoniae. FEMS Microbiol. Lett. 268:217-224.

11. Hanahan, D. 1983. Studies on transformation of Escherichia coli with plasmids. J. Mol. Biol. 166:557-580.

12. Hoskins, J., P. Matsushima, D.L. Mullen, J. Tang, G. Zhao, T.I. Meier, T.I. Nicas, and S.R. Jaskunas. 1999. Gene disruption studies of penicillin-binding proteins 1a, $1 \mathrm{~b}$ and $2 \mathrm{a}$ in Streptococcus pneumoniae. J. Bacteriol. 181:6552-6555.

13. Kell, C.M., U.K. Sharma, C.G. Dowson, C. Town, T.S. Balganesh, and B.G. Spratt. 1993. Deletion analysis of the essentiality of penicillin-binding proteins $1 \mathrm{~A}, 2 \mathrm{~B}$ and $2 \mathrm{X}$ of Streptococcus pneumoniae. FEMS Microbiol. Lett. 106:171-175.

14. Lacks, S., and R.D. Hotchkiss. 1960. A study of the genetic material determining an enzyme activity in pneumococcus. Biochim. Biophys. Acta 39:508-517. 
15. Land, A.D., H.C. Tsui, O. Kocaoglu, S.A. Vella, S.L. Shaw, S.K. Keen, L.T. Sham, E.E. Carlson, and M.E. Winkler. 2013. Requirement of essential Pbp2x and GpsB for septal ring closure in Streptococcus pneumoniae D39. Mol. Microbiol. 90:939-955.

16. Lanie, J.A., W.L. Ng, K.M. Kazmierczak, T.M. Andrzejewski, T.M. Davidsen, K.J. Wayne, H. Tettelin, J.I. Glass, and M.E. Winkler. 2007. Genome sequence of Avery's virulent serotype 2 strain D39 of Streptococcus pneumoniae and comparison with that of unencapsulated laboratory strain R6. J. Bacteriol. 189:38-51.

17. Macheboeuf, P., C. Contreras-Martel, V. Job, O. Dideberg and A. Dessen. 2006. Penicillin binding proteins: key players in bacterial cell cycle and drug resistance processes. FEMS Microbiol. Rev. 30:673-691.

18. Maestro, B., L. Novaková, D. Hesek, M. Lee, E. Leyva, S. Mobashery, J.M. Sanz, and P. Branny. 2011. Recognition of peptidoglycan and b-lactam antibiotics by the extracellular domain of the Ser/Thr protein kinase StkP from Streptococcus pneumoniae. FEBS Lett. 585: 357-363.

19. Mascher, T., M. Heintz, D. Zähner, M. Merai, and R. Hakenbeck. 2006. The CiaRH system of Streptococcus pneumoniae prevents lysis during stress induced by treatment with cell wall inhibitors and mutations in $p b p 2 x$ involved in beta-lactam resistance. J. Bacteriol. 188:1959-1968.

20. Massidda, O., L. Novakova, and W. Vollmer. 2013. From models to pathogens: how much have we learned about Streptococcus pneumoniae cell division? Environ. Microbiol. 15:3133-3157.

21. Maurer, P., B. Koch, I. Zerfaß, J. Krauß, M. van der Linden, J.-M. Frère, C. Contreras-Martel, and R. Hakenbeck. 2008. Penicillin-Binding Protein 2x of Streptococcus pneumoniae: Three new mutational pathways for remodelling an essential enzyme into a resistance determinant. J. Mol. Biol. 376:1403-1416.

22. Maurer, P., K. Todorova, J. Sauerbier and R. Hakenbeck. 2012. Mutations in Streptococcus pneumoniae penicillin-binding protein $2 \mathrm{x}$ : importance of the C-terminal penicillin-binding protein and serine/threonine kinaseassociated domains for beta-lactam binding. Microb. Drug Resist. 18:314-321.

23. Mir, M., J. Asong, X. Li, J. Cardot, G.J. Boons, and R.N. Husson. 2011. The extracytoplasmic domain of the Mycobacterium tuberculosis Ser/Thr kinase PknB binds specific muropeptides and is required for PknB localization. PLoS Pathog. 7:e1002182.

24. Morlot, C., A. Zapun, O. Dideberg, and T. Vernet. 2003. Growth and division of Streptococcus pneumoniae: localization of the high molecular weight penicillin-binding proteins during the cell cycle. Mol. Microbiol. 50:845-855.

25. Morlot, C., L. Bayle, M. Jacq, A. Fleurie, G. Tourcier, F. Galisson, T. Vernet, C. Grangeasse, and A.M. Di Guilmi. 2013. Interaction of Penicillin-Binding Protein 2x and Ser/Thr protein kinase StkP, two key players in Streptococcus pneumoniae R6 morphogenesis. Mol. Microbiol. 90:88-102.

26. Ottolenghi, E., and R.D. Hotchkiss. 1962. Release of genetic transforming agent from pneumococcal cultures during growth and disintegration. J. Exp. Med. 116:491-519.

27. Paik, J., I. Kern, R. Lurz, and R. Hakenbeck. 1999. Mutational analysis of the Streptococcus pneumoniae bimodular class A penicillin-binding proteins. J. Bacteriol. 181:3852-3856.
28. Pares, S., N. Mouz, Y. Pétillot, R. Hakenbeck, and O. Dideberg. 1996. X-ray structure of Streptococcus pneumoniae $\mathrm{PBP} 2 \mathrm{x}$, a primary penicillin target enzyme. Nat. Struct. Biol. 3:284-289.

29. Peters, K., I. Schweizer, K. Beilharz, C. Stahlmann, J.-W. Veening, R. Hakenbeck, and D. Denapaite. 2014. Streptococcus pneumoniae PBP2x mid-cell localization requires the $\mathrm{C}$-terminal PASTA domains and is essential for cell shape maintenance. Mol. Microbiol. [Epub ahead of print]; DOI: 10.1111/mmi.12588.

30. Salles, C., L. Creancier, J.P. Claverys, and V. Méjean. 1992. The high level streptomycin resistance gene from Streptococcus pneumoniae is a homologue of the ribosomal protein S12 gene from Escherichia coli. Nucleic Acids Res. 20:6103.

31. Sambrook, J., E.F. Fritsch, and T. Maniatis. 1989. MolecularCloning: A Laboratory Manual. Cold Spring Harbor Laboratory Press, Cold Spring Harbor, NY.

32. Sauvage, E., F. Kerff, M. Terrak, J. Ayala, and P. Charlier. 2008. The penicillin-binding proteins: structure and role in peptidoglycan biosynthesis. FEMS Microbiol. Rev. 32:234-258.

33. Shah, I.M., M.H. Laaberki, D.L. Popham, and J. Dworkin. 2008. A eukaryotic-like Ser/Thr kinase signals bacteria to exit dormancy in response to peptidoglycan fragments. Cell 135:486-496.

34. Sham, L.T., H.C. Tsui, A.D. Land, S.M. Barendt, and M.E. Winkler. 2012. Recent advances in pneumococcal peptidoglycan biosynthesis suggest new vaccine and antimicrobial targets. Curr. Opin. Microbiol. 15:194-203.

35. Squeglia, F., R. Marchetti, A. Ruggiero, R. Lanzetta, D. Marasco, J. Dworkin, M. Petoukhov, A. Molinaro, R. Berisio, and A. Silipo. 2011. Chemical basis of peptidoglycan discrimination by PrkC, a key kinase involved in bacterial resuscitation from dormancy. J. Am. Chem. Soc. 133:20676-20679.

36. Tsui, H.C., S.K. Keen, L.T. Sham, K.J. Wayne, and M.E. Winkler. 2011. Dynamic distribution of the SecA and SecY translocase subunits and septal localization of the HtrA surface chaperone/protease during Streptococcus pneumoniae D39 cell division. MBio 2:e00202-e00211.

37. Yeats, C., R.D. Finn, and A. Bateman. 2002. The PASTA domain: a beta-lactam-binding domain. Trends Biochem. Sci. 27:438-440.

38. Zapun, A., C. Contreras-Martel, and T. Vernet. 2008a. Penicillin-binding proteins and b-lactam resistance. FEMS Microbiol. Rev. 32:361-385.

39. Zapun, A., T. Vernet, and M.G. Pinho. 2008b. The different shapes of cocci. FEMS Microbiol. Rev. 32:345-360.

40. Zerfaß, I., R. Hakenbeck, and D. Denapaite. 2009. An important site in PBP2x of penicillin-resistant clinical isolates of Streptococcus pneumoniae: mutational analysis of Thr338. Antimicrob. Agents Chemother. 53:1107-1115.

Address correspondence to: Dalia Denapaite, $P h D$ Department of Microbiology University of Kaiserslautern Paul-Ehrlich Straße 23 D-67663 Kaiserslautern Germany

E-mail: denapait@rhrk.uni-kl.de 